\title{
ON DIMENSIONS OF FRAME SPECTRAL MEASURES AND THEIR FRAME SPECTRA
}

\author{
Ruxi Shi \\ Polish Academy of Sciences, Institute of Mathematics \\ ul. Śniadeckich 8, 00-656 Warszawa, Poland; rshi@impan.pl
}

\begin{abstract}
In this paper, we prove that the entropy dimension of a frame spectral measure is superior than or equal to the Beurling dimension of its frame spectrum.
\end{abstract}

\section{Introduction}

A set $\Lambda$ in a Hilbert space $\mathcal{H}$ is called a frame if there exist two constants $A, B>0$ such that for every $f \in \mathcal{H}$, we have

$$
A\|f\|^{2} \leq \sum_{\lambda \in \Lambda}|\langle f, \lambda\rangle|^{2} \leq B\|f\|^{2}
$$

where $\langle\cdot, \cdot\rangle$ is the inner product in $\mathcal{H}$. The constants $A$ and $B$ are called lower and upper bounds of the frame. Moreover, if only the upper bound hold in (1.1), then we call $\Lambda$ a Bessel set or Bessel sequence in $\mathcal{H}$. It is not hard to see that frame is a natural generalization of orthonormal basis (where $A=B=1$ ). If we restrict $\mathcal{H}=L^{2}(\mu)$ for some Borel measure $\mu$ on a locally compact abelian group $G$, then $\mu$ is called a frame spectral measure with frame spectrum $\Lambda$ if (1.1) holds and $\Lambda$ is contained in the dual group $\widehat{G}$, and furthermore called a spectral measure if $A=B=1$.

The notion of frame was introduced by Duffin and Schaeffer [2] in the context of nonharmonic Fourier series. Frames provide robust, basis-like (but non-unique) representations of vectors in a Hilbert space. The potential redundancy of frames often allows one to construct them more easily than bases, and to get better properties than those that are achievable using bases. Nowadays, frames have various applications in a wide range of areas. However, few properties of frame spectral measures are known. In this paper, we are interested in the relation between the dimensions of the frame spectral measure and its spectrum. It is believed that the "dimension" of the frame spectral measure should control the "dimension" of its spectrum. But only the case when the measure is self-similar was established $([3,8])$. In such case, the frame spectral measure is exact dimensional and its Hausdorff dimension controls the beurling dimension (see the definition in Section 2.2) of its frame spectrum. In general, it was conjectured in [8] that

Conjecture 1.1. If $\mu$ is a frame spectral measure with spectrum $\Lambda$ and compact support $T$ then $\operatorname{dim} \Lambda \leq \operatorname{dim}_{H} T$.

In this paper, we disprove Conjecture 1.1, which means that the Hausdorff dimension is not a candidate that controls the Berling dimension in general. Instead, we prove a similar version of Conjecture 1.1 by replacing Hausdorff dimension by upper entropy dimension (see its definition in Section 2.3). More precisely, supposing that $\mu$ is a frame spectral measure with frame spectrum $\Lambda$, we show that the

https://doi.org/10.5186/aasfm.2021.4629

2020 Mathematics Subject Classification: Primary 28A78, 42C05, 42B05, 94A17.

Key words: Beurling dimension, entropy dimension, frame spectral measure, frame spectrum. 
Beurling dimension of the frame spectrum $\Lambda$ is not superior than the upper entropy dimension of the frame spectral measure $\mu$, which allows one to see that the Beurling dimension and the upper entropy dimension are the proper notions of "dimensions" which are described above. Now we state our main result.

Theorem 1.2. Let $\mu$ be a Borel measure on $\mathbf{R}^{d}$. Suppose that $\mu$ is a frame spectral measure with frame spectrum $\Lambda$. Then we have

$$
\operatorname{dim} \Lambda \leq \overline{\operatorname{dim}_{e}} \mu,
$$

where $\operatorname{dim} \Lambda$ is the Beurling dimension of $\Lambda$ and $\overline{\operatorname{dim}_{e}} \mu$ is the upper entropy dimension of $\mu$.

The following theorem is formally stronger than but actually equivalent to Theorem 1.2 .

Theorem 1.3. Let $\mu$ be a Borel measure on $\mathbf{R}^{d}$. Suppose that $\mu$ is a frame spectral measure with frame spectrum $\Lambda$. Then we have

$$
\operatorname{dim} \Lambda \leq \inf _{\mu(K)>0, \mu(\partial K)=0} \overline{\operatorname{dim}_{e}} \mu_{K}
$$

where $\mu_{K}$ is the measure $\mu$ restricted on the Borel set $K$.

In fact, Theorem 1.2 is equivalent to Theorem 1.3 as follows: the necessity is trivial, and the sufficiency follows from Lemma 3.3 which states that if $\mu$ is a frame spectral measure then $\mu_{K}$ is also a frame spectral measure for every Borel set $K$ satisfying $\mu(\partial K)=0$.

In general, we can not expect that the equality holds in Theorem 1.2 or Theorem 1.3. We refer to the examples in [1] where a class of singular continuous measures are constructed, satisfying that the Beurling dimensions of their spectra are zero but their entropy dimensions are strictly positive.

Even though Theorem 1.3 is stated for frame spectral measures, we remark that only the upper bound in (1.1) plays a role in the proof of Theorem 1.3. In other words, if a Borel measure $\mu$ has a Bessel sequence $\Lambda$, then Theorems 1.2 and 1.3 hold for $\mu$ and $\Lambda$.

We organize our paper as follows. In Section 2, we recall several definitions of different dimensions, including Hausdorff dimension, entropy dimension, etc. In order to prove Theorem 1.2 in Section 4, we make some reduction of Theorem 1.2 in Section 3. In Section 5, we discuss the relation between Beurling dimension with other dimensions. Finally, in Section 6, we show some application of our main result.

\section{Preliminaries}

In this section, we recall several definitions of different dimensions.

2.1. Dyadic partitions. We first define the $n$-th dyadic partition of $\mathbf{R}$ by

$$
\mathcal{D}_{n}^{(1)}:=\left\{\left[\frac{k}{2^{n}}, \frac{k+1}{2^{n}}\right): k \in \mathbf{Z}\right\} .
$$

The $n$-th dyadic partition of $\mathbf{R}^{d}$ is then defined by

$$
\mathcal{D}_{n}^{(d)}:=\left\{I_{1} \times I_{2} \times \cdots \times I_{d}: I_{j} \in \mathcal{D}_{n}^{(1)}\right\} .
$$

If there is no confusion, we usually omit the superscript and write $\mathcal{D}_{n}$ for the $n$-th dyadic partition of $\mathbf{R}^{d}$. 
2.2. Dimensions of measure. Let $(X, \mathcal{B}, \mu)$ be a probability space. Let $\mathcal{A}$ be a partition of $X$. The Shannon entropy of $\mu$ with respect to $\mathcal{A}$ is defined by

$$
H(\mu, \mathcal{A})=\sum_{A \in \mathcal{A}}-\mu(A) \log \mu(A) .
$$

By convention the logarithm is taken in base 2 and $0 \log 0=0$. If the partition $\mathcal{A}$ is infinite, then the entropy $H(\mu, \mathcal{A})$ may be infinite.

Recall that $\mathcal{D}_{n}$ is the dyadic partition of $\mathbf{R}^{d}$ with diameter $2^{-n}$. The entropy dimension of $\mu$ is defined by the formula

$$
\operatorname{dim}_{\mathrm{e}} \mu=\lim _{n \rightarrow \infty} \frac{1}{n} H\left(\mu, \mathcal{D}_{n}\right),
$$

if the limit exists (otherwise we take limsup or liminf as appropriate, denoted by $\overline{\operatorname{dim}}_{e} \mu$ and $\underline{\operatorname{dim}}_{e} \mu$ respectively).

The lower Hausdorff dimension of $\mu$ is defined by

$$
\underline{\operatorname{dim}}_{H} \mu=\inf \left\{\operatorname{dim}_{H} A: \mu(A)>0\right\},
$$

and the upper Hausdorff dimension of $\mu$ is defined by

$$
\overline{\operatorname{dim}}_{H} \mu=\inf \left\{\operatorname{dim}_{H} A: \mu(A)=1\right\} .
$$

Here $\operatorname{dim}_{H} A$ is the Hausdorff dimension of $A$. In what follows, the open ball of radius $r$ centered at $x$ is denoted by $B(x, r)$. A measure $\mu$ is exact dimensional if the local dimension

$$
\lim _{r \rightarrow 0} \frac{\log \mu(B(x, r))}{\log r}
$$

exists and is $\mu$-a.e. constant, which is denoted by $\operatorname{dim} \mu$. Here and in what follows, we denote by $\mathcal{P}\left(\mathbf{R}^{d}\right)$ the space of probability measures on $\mathbf{R}^{d}$. The following lemma is well-known and its proof can be found, for example, in [5, Theorem 1.1].

Lemma 2.1. If $\mu \in \mathcal{P}\left(\mathbf{R}^{d}\right)$ is exact dimensional, then $\operatorname{dim}_{e} \mu$ exists and is equal to $\operatorname{dim} \mu$.

2.3. Beurling dimension of countable sets. Let $\Lambda$ be a countable set in $\mathbf{R}^{d}$. For $r>0$, the upper Beurling density corresponding to $r$ (or $r$-Beurling density) of $\Lambda$ is defined by the formula

$$
\mathfrak{D}_{r}^{+}:=\limsup _{h \rightarrow \infty} \sup _{x \in \mathbf{R}^{d}} \frac{\sharp(\lambda \cap B(x, h))}{h^{r}} .
$$

The (upper) Beurling dimension of $\Lambda$ is defined by

$$
\operatorname{dim} \Lambda=\sup \left\{r>0: \mathfrak{D}_{r}^{+}(\Lambda)>0\right\},
$$

or alternatively,

$$
\operatorname{dim} \Lambda=\inf \left\{r>0: \mathfrak{D}_{r}^{+}(\Lambda)<+\infty\right\} .
$$

A basic property of Beurling dimension is that $\operatorname{dim} s \Lambda=\operatorname{dim} \Lambda$ for all $s \in \mathbf{R} \backslash\{0\}$.

\section{Reduction of the main result}

In this section, our goal is to make some reduction of Theorem 1.2.

The following lemma is a direct consequence of the definition of frame spectral measures. We omit the proof and leave the readers to work out the details.

Lemma 3.1. Let $\mu$ be a frame spectral measure in $\mathbf{R}^{d}$ with spectrum $\Lambda$ and frame bounds $0<A \leq B<\infty$. Then we have the following properties. 
(1) For any $v, t \in \mathbf{R}^{d}, \mu(\cdot+v)$ is a frame spectral measure with spectrum $\Lambda+t$ and frame bounds $A, B$.

(2) For any non-zero $c \in \mathbf{R}, c \cdot \mu$ is a frame spectral measure with spectrum $\Lambda$ and frame bounds $c A, c B$.

(3) For any non-zero $s \in \mathbf{R}, \mu(\cdot \times s)$ is a frame spectral measure with spectrum $s \Lambda$ and frame bounds $A, B$.

We observe that if putting $\mathcal{H}=L^{2}(\mu)$ and $f=\bar{\lambda}^{-1}$ for some $\lambda \in \Lambda$ in (1.1), then we have $\mu\left(\mathbf{R}^{d}\right)<\infty$. Since $c \cdot \mu$ is also a frame spectral measure for any $c \in \mathbf{R}$, we might assume that $\mu \in \mathcal{P}\left(\mathbf{R}^{d}\right)$.

It is well known that $[13,9]$ that if $\mu$ is a frame spectral measure with frame $\Lambda$, then it has to be of "pure type".

Theorem 3.2. [9, Theorem 1.1, Proposition 2.1] Let $\mu$ be a frame spectral measure with frame $\Lambda$. Then $\mu$ is either discrete with $\sharp \Lambda<+\infty$, absolutely continuous with $\mathfrak{D}_{d}^{-}(\Lambda)>0$ or singular continuous with $\mathfrak{D}_{d}^{-}(\Lambda)=0$.

If $\mu$ is discrete, then it has finitely many atoms [9], implying that $\operatorname{dim} \Lambda=$ $\operatorname{dim}_{e} \mu=0$. If $\mu$ is absolutely continuous, then it is supported on a set of finite Lebesgue measure in $\mathbf{R}^{d}$, and its density function is bounded from above and from below almost everywhere on the support [14]. It follows that if $\mu$ is absolutely continuous, then $\operatorname{dim} \Lambda=\operatorname{dim}_{e} \mu=d$. By the above argument, it is sufficient to prove Theorem 1.2 for singular continuous measures.

Let $\mu \in \mathcal{P}\left(\mathbf{R}^{d}\right)$. For a Borel set $K \subset \mathbf{R}^{d}$, we denote by

$$
\mu_{K}(\cdot):=\mu(\cdot \cap K),
$$

the measure $\mu$ restricted on $K$. Moreover, if $K$ is a dyadic cube in $[0,1]^{d}$ with $\mu(K)>0$, we denote by

$$
\mu_{K}^{\square}(\cdot):=\frac{1}{\mu(K)}\left(S_{K}\right)_{*} \mu_{K}(\cdot),
$$

where $S_{K}$ is the affine bijective map from $K$ to $[0,1]^{d}$ and $\left(S_{K}\right)_{*} \mu_{K}(\cdot)$ is the pushforward of $\mu_{K}$, i.e. the measure $\mu_{K}\left(S_{K}^{-1}(\cdot)\right)$. Obviously, we have $\mu_{K}^{\square} \in \mathcal{P}\left([0,1]^{d}\right)$.

The following lemma provides that the restriction of a frame spectral measure is also a frame spectral measure. A general version of the following lemma can be found in [7]. We include the proof here for completeness.

Lemma 3.3. Let $\mu$ be a frame spectral measure on $\mathbf{R}^{d}$. Let $K \subset \mathbf{R}^{d}$ be a Borel subset satisfying that $\mu(\partial K)=0$. Then $\mu_{K}$ is also a frame spectral measure having the same spectrum and frame bounds with the measure $\mu$.

Proof. For any $f \in L^{2}\left(\mu_{K}\right)$, we extend $f$ into the space $L^{2}(\mu)$ by taking $f(x)=0$ for any $x$ outside $\bar{K}$. Since $\mu_{K^{c}}(\bar{K})=0$, we have $\langle f, g\rangle_{\mu_{K^{c}}}=0$ for all $g \in L^{2}(\mu)$. It follows that $\langle f, g\rangle_{\mu}=\langle f, g\rangle_{\mu_{K}}$ for all $g \in L^{2}(\mu)$, and in particular $\|f\|_{\mu}=\|f\|_{\mu_{K}}$. Thus we conclude that $\mu_{K}$ is also a frame spectral measure and has the same spectrum and frame bounds with the measure $\mu$.

Remark. After this paper was completed, thanks to Zhiqiang Wang, we realized that the condition $\mu(\partial K)=0$ in Lemma 3.3 is not really needed in the proof. Indeed, since the set $K$ is Borel, we can extend any function from $L^{2}\left(\mu_{K}\right)$ to $L^{2}(\mu)$ by taking 0 outside $K$. Then the extended function is also Borel measurable and the conclusion in Lemma 3.3 remains the same. As a consequence, we could drop the condition $\mu(\partial K)=0$ in Theorem 1.3 and simplify the proof of Theorem 1.3. 
The following lemma shows that we can find a unit cube satisfying the condition (of $K$ ) in Lemma 3.3 .

Lemma 3.4. Let $\mu \in \mathcal{P}\left(\mathbf{R}^{d}\right)$. Then there exists a unit cube $v+[0,1]^{d}$ for some $v \in \mathbf{R}^{d}$ such that $\mu\left(v+[0,1]^{d}\right)>0$ and $\mu\left(\partial\left(v+[0,1]^{d}\right)\right)=0$.

Proof. Since $\mu \in \mathcal{P}\left(\mathbf{R}^{d}\right)$, there exists $u \in \mathbf{R}^{d}$ such that $\mu\left(u+[0,1 / 2]^{d}\right)>0$. Let $u_{t}=u-(t, t, \ldots, t)$. Since $u_{t}+[0,1]^{d}$ contains $u+[0,1 / 2]^{d}$ for any $t \in\left[0, d^{1 / 2} / 2\right]$, we see that $\mu\left(u_{t}+[0,1]^{d}\right)>0$. Observe that $\partial[0,1]^{d}=A \cup B$ where $A=\{x=$ $\left.\left(x_{i}\right)_{1 \leq i \leq d}: x_{i} \in[0,1], \exists x_{j}=0\right\}$ and $B=\left\{x=\left(x_{i}\right)_{1 \leq i \leq d}: x_{i} \in[0,1], \exists x_{j}=1\right\}$. Moreover, it is easy to check that $\left(u_{t}+A\right) \cap\left(u_{s}+A\right)=\emptyset$ and $\left(u_{t}+B\right) \cap\left(u_{s}+B\right)=\emptyset$ for distinct $s, t \in\left[0, d^{1 / 2} / 2\right]$. By the fact that the sum of an uncountable number of positive numbers is infinite ${ }^{1}$, we get that $\mu\left(u_{t}+A\right)>0\left(\operatorname{resp} . \mu\left(u_{t}+B\right)>0\right)$ for at most countably many $t \in\left[0, d^{1 / 2} / 2\right]$. Thus there exists $t \in\left[0, d^{1 / 2} / 2\right]$ such that $\mu\left(u_{t}+A\right)=\mu\left(u_{t}+B\right)=0$ and consequently $\mu\left(\partial\left(u_{t}+[0,1]^{d}\right)\right)=0$.

Let $v$ be as in Lemma 3.4. Then by Lemma 3.1 and Lemma 3.3, the measure

$$
\frac{1}{\mu\left(v+[0,1]^{d}\right)} \mu_{v+[0,1]^{d}}(\cdot+v) \in \mathcal{P}\left([0,1]^{d}\right)
$$

has the same spectrum with $\mu$. Since

$$
\overline{\operatorname{dim}}_{e} \frac{1}{\mu\left(v+[0,1]^{d}\right)} \mu_{v+[0,1]^{d}}(\cdot+v) \leq \overline{\operatorname{dim}}_{e} \mu,
$$

it is sufficient to prove Theorem 1.2 for measures in $\mathcal{P}\left([0,1]^{d}\right)$.

The following lemma shows that the dyadic partition is "nice" up to a scaling of $\mu$.

Lemma 3.5. Let $\mu \in \mathcal{P}\left([0,1]^{d}\right)$. Then there exists $1 \leq s<\infty$ such that $\mu(\cdot \times s) \in \mathcal{P}\left([0,1]^{d}\right)$ satisfies that $\mu((\cdot \times s) \cap \partial D)=0$ for all $D \in \mathcal{D}_{n}$ where $\left\{\mathcal{D}_{n}\right\}_{n=1}^{\infty}$ is the set of the dyadic partitions.

Proof. Fix $n \in \mathbf{N}$ and $D \in \mathcal{D}_{n}$. Suppose $D=u+\left[0,2^{-n}\right]^{d}$ where $u=\left(u_{i}\right)_{1 \leq i \leq d} \in$ $[0,1]^{d}$. We decompose $\partial D=A \cup B$ where $A=\left\{x=\left(x_{i}\right)_{1 \leq i \leq d}: x_{i} \in\left[u_{i}, u_{i}+\right.\right.$ $\left.\left.2^{-n}\right], \exists x_{j}=u_{j}\right\}$ and $B=\left\{x=\left(x_{i}\right)_{1 \leq i \leq d}: x_{i} \in\left[u_{i}, u_{i}+2^{-n}\right], \exists x_{j}=u_{j}+2^{-n}\right\}$. it is easy to check that $t A \cap s A=\emptyset$ and $t B \cap s B=\emptyset$ for distinct $s, t \in[1, \infty)$. By the fact that the sum of an uncountable number of positive numbers is infinite (See Footnote 1 ), we get that $\mu(t A)>0$ (resp. $\mu(t B)>0$ ) for at most countably many $t \in[1, \infty)$. It follows that $\mu(t \partial D)>0$ for at most countably many $t \in[1, \infty)$. Since $\left\{\mathcal{D}_{n}\right\}_{n=1}^{\infty}$ consists of countably many elements, there exists $1 \leq s<\infty$ such that $\mu(\cdot \times s)$ satisfies that $\mu((\cdot \times s) \cap \partial D)=0$ for all $D \in \mathcal{D}_{n}$.

By Lemma 3.5, Lemma 3.1 (3) and the facts that $\operatorname{dim} s \Lambda=\operatorname{dim} \Lambda$ and $\overline{\operatorname{dim}}_{e} \mu(\cdot \times$ $s)=\overline{\operatorname{dim}}_{e} \mu$, we might assume $s=1$ for the sake of simplicity.

Finally, we summarize the reductions made so far in the following list.

Reduction 3.6. In order to prove Theorem 1.2, we might assume that a frame spectral measure $\mu$ in $\mathbf{R}^{d}$ has the following structure:

(1) The measure $\mu$ is singular continuous.

(2) The measure $\mu$ belongs to $\mathcal{P}\left([0,1]^{d}\right)$.

(3) The dyadic partitions $\left\{\mathcal{D}_{n}\right\}_{n=1}^{\infty}$ satisfy that $\mu(\partial D)=0$ for all $D \in \mathcal{D}_{n}$.

\footnotetext{
${ }^{1}$ Suppose that $I$ is an uncountable set and $a_{i}>0$ for $i \in I$. Let $C_{n}=\left\{a_{i}: a_{i}>1 / n, i \in I\right\}$. Then there exists $n \in \mathbf{N}$ such that $C_{n}$ contains infinite elements. Thus $\sum_{i \in I} a_{i} \geq \sum_{a_{i} \in C_{n}} a_{i} \geq$ $\sum_{a_{i} \in C_{n}} 1 / n=+\infty$.
} 


\section{Proof of main result}

Let $\mu \in \mathcal{P}\left([0,1]^{d}\right)$. It is well-known that the dual group $\widehat{\mathbf{R}^{d}}$ consists of exponential functions which is isomorphic to $\mathbf{R}^{d}$. We could thus identify $\mathbf{R}^{d}$ with the subspace in $L^{2}(\mu)$ and write $\langle f, \lambda\rangle_{\mu}$ for $f \in L^{2}(\mu)$ and $\lambda \in \mathbf{R}^{d}$. More precisely, we write the inner products

$$
\langle f, \lambda\rangle_{\mu}=\int_{[0,1]^{d}} f(x) e^{-2 \pi i \lambda \cdot x} d \mu(x)
$$

and

$$
\langle t, \lambda\rangle_{\mu}=\int_{[0,1]^{d}} e^{2 \pi i(t-\lambda) \cdot x} d \mu(x),
$$

for $f \in L^{2}(\mu)$ and $t, \lambda \in \mathbf{R}^{d}$.

The following two lemmas not only has its own interest but also are useful to prove our main result.

Lemma 4.1. Suppose that the measure $\mu \in \mathcal{P}\left([0,1]^{d}\right)$ is a frame spectral measure with spectrum $\Lambda$ and frame bounds $0<A \leq B<\infty$. Let $n>0$. Then for any $D \in \mathcal{D}_{n}$ with $\mu(D)>0$, and for any $t \in \mathbf{R}^{d}$, we have

$$
\frac{A}{\mu(D)} \leq \sum_{\lambda \in \Lambda}\left|\left\langle\frac{1}{2^{n}} t, \frac{1}{2^{n}} \lambda\right\rangle_{\mu_{D}^{\square}}\right|^{2} \leq \frac{B}{\mu(D)} .
$$

Proof. Let $D \in \mathcal{D}_{n}$ with $\mu(D)>0$. Let $t \in \mathbf{R}^{d}$. By Lemma 3.3 and Reduction 3.6 (3), we have

$$
A \mu(D) \leq \sum_{\lambda \in \Lambda}\left|\langle t, \lambda\rangle_{\mu_{D}}\right|^{2} \leq B \mu(D)
$$

For any $\lambda \in \Lambda$, we observe that

$$
\langle t, \lambda\rangle_{\mu_{D}}=\mu(D) e^{2 \pi i(t-\lambda) \cdot v(D)}\left\langle\frac{1}{2^{n}} t, \frac{1}{2^{n}} \lambda\right\rangle_{\mu_{D}^{\square}},
$$

where $v(D) \in \mathbf{R}^{d}$ is the vector satisfying $D=v(D)+\left[0, \frac{1}{2^{n}}\right]^{d}$. It follows that

$$
\left|\langle t, \lambda\rangle_{\mu_{D}}\right|=\mu(D)\left|\left\langle\frac{1}{2^{n}} t, \frac{1}{2^{n}} \lambda\right\rangle_{\mu_{D}^{\square}}\right|,
$$

for any $\lambda \in \Lambda$. Combing (4.1) and (4.2), we complete the proof.

Lemma 4.2. Let $\mu \in \mathcal{P}\left(\mathbf{R}^{d}\right)$. Then for any $0<\epsilon<1$, there exists $\delta=\delta(\epsilon)>0$ such that for any $|\xi|<\delta$ and for any $D \in \mathcal{D}_{n}$ with $\mu(D)>0$, we have

$$
\left|\widehat{\mu_{D}^{\square}}(\xi)\right|>\epsilon .
$$

Proof. Let $0<\epsilon<1$. Pick arbitrary $D \in \mathcal{D}_{n}$. A simple computation shows that

$$
\left|\widehat{\mu_{D}^{\square}}(\xi)\right|=\left|\int_{[0,1]^{d}} e^{2 \pi i \xi \cdot x} d \mu_{D}^{\square}(x)\right| \geq\left|\int_{[0,1]^{d}} \cos (2 \pi \xi \cdot x) d \mu_{D}^{\square}(x)\right| .
$$

We choose $0<\delta<\frac{1}{4 d}$ small enough such that $\cos (2 d \pi \delta)>\epsilon$. Since $\cos (\theta)$ is positive and decreasing for $\theta \in\left(0, \frac{\pi}{2}\right)$, we have $\cos (2 d \pi \theta)>\epsilon$ for all $0<\theta<\delta$. Then for any $|\xi|<\delta$ and $x \in[0,1]^{d}$, we have that $|\xi \cdot x|<d \delta$ and consequently that $\cos (2 \pi \xi \cdot x)>\epsilon$. It follows from (4.3) that

$$
\left|\widehat{\mu_{D}^{\square}}(\xi)\right|>\epsilon .
$$


This completes the proof.

Now we prove our main result.

Proof of Theorem 1.2. Pick arbitrary $s>0$. Then there exists $N>0$ such that for any $n>N$, we have

$$
\overline{\operatorname{dim}}_{e} \mu+s \geq \frac{1}{n} \sum_{D \in \mathcal{D}_{n}}-\mu(D) \log \mu(D) .
$$

It follows that

$$
\left(2^{n}\right)^{\overline{\operatorname{dim}}_{e} \mu+s} \geq 2^{\sum_{D \in \mathcal{D}_{n}}-\mu(D) \log \mu(D)}=\prod_{D \in \mathcal{D}_{n}, \mu(D)>0} \mu(D)^{-\mu(D)} .
$$

Let $h>2^{N}$. Then there exists a positive integer $n_{h}$ such that $2^{n_{h}-1}<h \leq 2^{n_{h}}$. Let $\epsilon>0$. Let $\delta=\delta(\epsilon)$ which is defined in Lemma 4.2. Let $\rho$ be the minimal integer such that $2^{-\rho}<\delta$. For any $t \in \mathbf{R}^{d}$ and any $D \in \mathcal{D}_{n_{h}+\rho}$, we have

$$
\begin{aligned}
\epsilon^{2} \cdot \sharp(\Lambda \cap B(t, h)) & \leq \sum_{\lambda \in \Lambda \cap B(t, h)}\left|\left\langle\frac{1}{2^{n_{h}+\rho}} t, \frac{1}{2^{n_{h}+\rho}} \lambda\right\rangle_{\mu_{D}^{\square}}\right|^{2} \\
& \leq \sum_{\lambda \in \Lambda}\left|\left\langle\frac{1}{2^{n_{h}+\rho}} t, \frac{1}{2^{n_{h}+\rho}} \lambda\right\rangle_{\mu_{D}^{\square}}\right|^{2} \leq \frac{B}{\mu(D)} .
\end{aligned}
$$

Since $\sum_{D \in \mathcal{D}_{n_{h}+\rho}} \mu(D)=1$ and (4.5) holds for all $D \in \mathcal{D}_{n_{h}+\rho}$ with $\mu(D)>0$, we have

$$
\begin{aligned}
\sharp(\Lambda \cap B(t, h)) & =\prod_{D \in \mathcal{D}_{n_{h}+\rho, \mu(D)>0}}(\sharp(\Lambda \cap B(t, h)))^{\mu(D)} \\
& \leq B \epsilon^{-2} \prod_{D \in \mathcal{D}_{n_{h}+\rho, \mu(D)>0}} \mu(D)^{-\mu(D)}
\end{aligned}
$$

It follows from (4.4) and (4.6) that

$$
\frac{\sharp(\Lambda \cap B(t, h))}{h^{\overline{\operatorname{dim}}_{e} \mu+s}} \leq \frac{\sharp(\Lambda \cap B(t, h))}{2^{\left(n_{h}-1\right)\left(\overline{\operatorname{dim}}_{e} \mu+s\right)}} \leq B \epsilon^{-2} \cdot 2^{(1+\rho)\left(\overline{\operatorname{dim}}_{e} \mu+s\right)} .
$$

Then we deduce that $\operatorname{dim} \Lambda \leq \overline{\operatorname{dim}}_{e} \mu+s$. Since $s$ can be chosen arbitrarily close to 0 , we conclude that $\operatorname{dim} \Lambda \leq \overline{\operatorname{dim}}_{e} \mu$.

\section{Further discussion}

In this section, we will discuss several different notions of dimensions (or conditions) and its relations with Beurling dimension and entropy dimension.

5.1. Lev's condition. Let $\mu \in \mathcal{P}\left(\mathbf{R}^{d}\right)$. Given a real number $\alpha$ with $0 \leq \alpha \leq d$. Lev considered the following condition in [15]:

$$
\liminf _{r \rightarrow \infty} \frac{1}{r^{d-\alpha}} \int_{-r}^{r}|\widehat{\mu}(t)|^{2} d t>0
$$

He proved that if a frame spectral measure $\mu$ with frame spectrum $\Lambda$ satisfies (5.1), then

$$
\sup _{x \in \mathbf{R}^{d}} \sharp(\Lambda \cap B(x, r)) \leq C r^{\alpha},
$$

for some constant $C$ which does not depend on $r$. We define

$$
L(\mu)=\inf \{\alpha:(5.1) \text { holds for } \mu \text { and } \alpha\} \text {. }
$$


Therefore we restate (5.2) as follows.

Lemma 5.1. Let $\mu$ be a frame spectral measure with frame spectrum $\Lambda$. Then we have

$$
\operatorname{dim} \Lambda \leq L(\mu)
$$

The value $L(\mu)$ is sometimes related to the "dimension" of $\mu$. For example, if $\mu$ is a certain self-similar measure with Hausdorff dimension $\alpha$, then $L(\mu)=\alpha$. However, such relation is very difficult to establish and compute in some cases. For instant, as far as I know, the condition (5.1) is unknown for self-affine measures. On the other hand, we will see that the entropy dimension is well established for self-affine measures in the next section.

5.2. Hausdorff dimension. For a Borel measure $\mu$, we know the facts that $\underline{\operatorname{dim}}_{H} \mu \leq \overline{\operatorname{dim}}_{e} \mu$ and that $\overline{\operatorname{dim}}_{H} \mu$ is not comparable with $\overline{\operatorname{dim}}_{e} \mu$ (see for example [5]). A natural question arises as to whether the entropy dimension can be replaced by the Hausdorff dimension in Theorems 1.2 and 1.3. We will give a negative answer to this question in the following.

Let $I \subset \mathbf{N}$ and $p$ be a prime number. For $n \in \mathbf{N}$, let

$$
I_{n}=\{i \in I: i \leq n\}
$$

be the finite subset of $I$ and let

$$
C\left(I_{n}\right)=\left\{\sum_{i \in I_{n}} b_{i} p^{-i}: b_{i} \in\{0,1, \cdots, p-1\}\right\}
$$

be the finite subset of the unit interval $[0,1]$. It is not hard to see that the weak limit of $\frac{1}{\sharp C\left(I_{n}\right)} \delta_{C\left(I_{n}\right)}$ exists, which is denoted by $\nu_{I}$, as $n$ tends to infinity. Let

$$
\Lambda_{I_{n}}=\left\{\sum_{i \in I_{n}} b_{i} p^{i}: b_{i} \in\{0,1, \cdots, p-1\}\right\}
$$

be the finite subset of $\mathbf{Z}$. Obviously, we have the inclusion $\Lambda_{I_{1}} \subset \Lambda_{I_{2}} \subset \cdots$. Let

$$
\Lambda_{I}=\bigcup_{i \in \mathbf{N}} \Lambda_{I_{n}}
$$

In [16], the author showed that the measure $\nu_{I}$ is a spectral measure with spectrum $\Lambda_{I}$. Moreover, it is computed that

$$
\operatorname{dim} \Lambda_{I}=\overline{\operatorname{dim}}_{e} \nu_{I}=\limsup _{n \rightarrow \infty} \frac{\sharp I_{n}}{n},
$$

and

$$
\operatorname{dim}_{H} \operatorname{supp}(\mu)=\overline{\operatorname{dim}}_{H} \nu_{I}=\underline{\operatorname{dim}}_{H} \nu_{I}=\liminf _{n \rightarrow \infty} \frac{\sharp I_{n}}{n} .
$$

At the same time, it is shown that

$$
\overline{\operatorname{dim}}_{e} \nu_{I}=\inf _{\mu(K)>0, \mu(\partial K)=0}\left\{\overline{\operatorname{dim}}_{e}\left(\nu_{I}\right)_{K}\right\},
$$

and

$$
\begin{aligned}
\operatorname{dim}_{H} \operatorname{supp}(\mu) & =\inf _{\mu(K)>0, \mu(\partial K)=0}\left\{\overline{\operatorname{dim}}_{H}\left(\nu_{I}\right)_{K}\right\} \\
& =\inf _{\mu(K)>0, \mu(\partial K)=0}\left\{\underline{\operatorname{dim}}_{H}\left(\nu_{I}\right)_{K}\right\}
\end{aligned}
$$


It is not hard to pick suitable $I \subset \mathbf{N}$ such that

$$
\liminf _{n \rightarrow \infty} \frac{\sharp I_{n}}{n}<\limsup _{n \rightarrow \infty} \frac{\sharp I_{n}}{n} .
$$

Under the condition (5.3), we have

$$
\operatorname{dim} \Lambda_{I}=\overline{\operatorname{dim}}_{e} \nu_{I}>\operatorname{dim}_{H} \operatorname{supp}(\mu)=\overline{\operatorname{dim}}_{H} \nu_{I}=\underline{\operatorname{dim}}_{H} \nu_{I} .
$$

This disproves Conjecture 1.1.

5.3. Fourier dimension. A lower bound of Beurling dimension was obtained in Theorem 1.3 [12] that if $\mu$ is a frame spectral measure with spectrum $\Lambda$, then

$$
\operatorname{dim} \Lambda \geq \operatorname{dim}_{F} \mu,
$$

where $\operatorname{dim}_{F}$ is the Fourier dimension which is defined by the formula

$$
\operatorname{dim}_{F} \mu:=\sup \left\{0 \leq s \leq d: \exists C, \forall \xi,|\widehat{\mu}(\xi)| \leq C|\xi|^{-s / 2}\right\} .
$$

Combining this with Lemma 3.3, we obtain the following theorem.

Theorem 5.2. Let $\mu$ be a Borel measure on $\mathbf{R}^{d}$. Suppose that $\mu$ is a frame spectral measure with frame spectrum $\Lambda$. Then we have

$$
\operatorname{dim} \Lambda \geq \sup _{\mu(K)>0, \mu(\partial K)=0} \operatorname{dim}_{F} \mu_{K} .
$$

A direct consequence of Theorems 1.3 and 5.2 is the following necessary condition for frame spectral measures.

Corollary 5.3. Let $\mu$ be a Borel measure on $\mathbf{R}^{d}$. Suppose that $\mu$ is a frame spectral measure with frame spectrum $\Lambda$. Then we have

$$
\sup _{\mu(K)>0, \mu(\partial K)=0} \operatorname{dim}_{F} \mu_{K} \leq \inf _{\mu(K)>0, \mu(\partial K)=0} \overline{\operatorname{dim}_{e}} \mu_{K} .
$$

Using Corollary 5.3, some non frame spectral measures could be shown as follows.

Corollary 5.4. Let $\mu, \nu$ and $\rho$ be Borel measures on $\mathbf{R}^{d}$. Suppose that $\operatorname{dim}_{F} \mu>$ $\overline{\operatorname{dim}}_{e} \nu$ and $\mu(\operatorname{supp}(\nu+\rho))=\nu(\operatorname{supp}(\mu+\rho))=0$. Then the measure $\mu+\nu+\rho$ is not a frame spectral measure.

We end up this section by proposing some open questions. We remark that if $\mu$ is absolutely continuous or discrete, then the equality holds in (5.5) in Corollary 5.3. Hence we might ask the same question for singular continuous measures:

Question 5.5. Does there exist a frame spectral measure which is singular continuous and the equality holds in (5.5)?

As far as I know, we don't yet have an example of frame spectral measures that have non-zero Fourier dimension. Thus we might ask the following question.

Question 5.6. Does there exist a frame spectral measure that is singular continuous and has non-zero Fourier dimension?

\section{Potential examples}

In this section, we apply Theorems 1.2 and 1.3 for various measures. Since few concrete examples of frame spectral measures are known, the results in this section might be helpful to find new examples of measures of different type.

6.1. Self-affine measures. A function $\varphi$ is called a contraction on a complete metric space $X$ with metric $d$ if $d(\varphi(x), \varphi(y))<d(x, y)$ holds for every $x \neq y \in$ 
$X$. If $\left\{\varphi_{i}\right\}_{1 \leq i \leq N}$ are contractions of $X$ it is well-known that there exists a unique non-empty compact set $K \subset X$ such that $K=\bigcup_{1<i<N} \varphi_{i}(K)$ (see [11]). In this circumstance the tuple $\left\{\varphi_{i}\right\}_{1 \leq i \leq N}$ is called an iterated function system (IFS) and $F$ its attractor. A central problem in the study of iterated function systems is to calculate or estimate the dimension of the attractor $F$ for various notions of fractal dimension, most especially the Hausdorff dimension. Particular interest has been given to the case of affine iterated function system, where the ambient space $X$ is given by $\mathbf{R}^{d}$ and the contractions $\varphi_{i}$ take the form $\varphi_{i}: x \mapsto A_{i} x+b_{i}$ for certain (usually invertible) linear maps $A_{i} \in \mathcal{L}\left(\mathbf{R}^{d}, \mathbf{R}^{d}\right)$ and vectors $b_{i}$. The associated attractors are called self-affine.

Very recently, Hochman and Rapaport [10] proved that if $\mu$ is a self-affine measure in the plane whose defining IFS acts totally irreducibly and satisfies an exponential separation condition, then its dimension is equal to its Lyapunov dimension. Applying Theorem 1.2, we get the following corollary.

Corollary 6.1. Let $\Phi=\left\{\varphi_{i}\right\}_{i \in J}$ be a finite system of invertible affine contractions of $\mathbf{R}^{2}$. Suppose that $\Phi$ has no common fixed point, satisfies the non-conformality and total irreducibility assumptions, and is exponentially separated. Let $p$ be a positive probability vector. Let $\mu=\sum p_{i} \cdot \varphi_{i} \mu$ be the associated self-affine measure. Assume that $\mu$ is a frame spectral measure with frame spectrum $\Lambda$. Then

$$
\operatorname{dim} \Lambda \leq \min \left\{2, \operatorname{dim}_{L} \mu\right\},
$$

where $\operatorname{dim}_{L}$ stands for Lyapunov dimension.

In general, Feng [6] proved that every ergodic invariant measure for an affine IFS is exact dimensional, and its Hausdorff dimension satisfies a Ledrappier-Young type formula. Applying Theorem 1.2, we have the following corollary.

Corollary 6.2. Let $\mu$ be an ergodic invariant measure for an affine IFS. Suppose that $\mu$ is a frame spectral measure with frame spectrum $\Lambda$. Then we have

$$
\operatorname{dim} \Lambda \leq \operatorname{dim} \mu .
$$

6.2. $\times \boldsymbol{\beta}$-invariant measures. Let $T_{\beta}$ be the multiplication by $\beta$ modulo one on the unite interval. It is well known that if $\beta$ is Pisot number and $\mu$ is $T_{\beta^{-}}$ invariant, then $\mu$ is exact dimensional. Thus we have the following corollary as a direct consequence of Theorem 1.2.

Corollary 6.3. Let $\beta$ be a Pisot number. If $\mu$ is a $T_{\beta}$-invariant frame spectral measure with spectrum $\Lambda$, then we have

$$
\operatorname{dim} \Lambda \leq \operatorname{dim} \mu .
$$

6.3. Measures of "mixed type". It is used to be conjectured that a pure type phenomenon should also exist within the class of singular continuous measures, that is to say, all frame spectral measures are exact dimensional. The first counterexample was constructed by Lev [15] as follows.

Let $\mu \in \mathcal{P}\left(\mathbf{R}^{n}\right)$ and $\nu \in \mathcal{P}\left(\mathbf{R}^{m}\right)$. We define a new measure $\rho$ on $\mathbf{R}^{n+m}$ by

$$
\rho=\mu \times \delta_{0}+\delta_{0} \times \nu,
$$

where $\delta_{0}$ denotes the Dirac measure at the origin. It is not hard to see that $\rho$ is the singular measure whose support is contained in $\left(\mathbf{R}^{n} \times\{0\}\right) \cup\left(\{0\} \times \mathbf{R}^{m}\right)$. The frame spectral measure of "mixed type" is constructed in the following theorem. 
Theorem 6.4. [15, Theorem 2.1] Assume that two measures $\mu$ and $\nu$ are continuous frame spectral measures. Then the measure $\rho$ defined above is also a frame spectral measure.

Applying Theorem 1.2 to the measure $\rho$ of "mixed type" in Theorem 6.4, we have the following corollary.

Corollary 6.5. Let $\mu, \nu, \rho$ be defined in Theorem 6.4. Suppose that $\Lambda$ is a frame spectrum of $\rho$. Then we have

$$
\operatorname{dim} \Lambda \leq \min \left\{\overline{\operatorname{dim}}_{e} \mu, \overline{\operatorname{dim}}_{e} \nu\right\}
$$

Acknowledgments. We would like to thank Chun-Kit Lai for many valuable remarks. We are also grateful for the anonymous referees for their valuable suggestions and comments. This work was partially supported by the Centre of Excellence in Analysis and Dynamics Research funded by the Academy of Finland.

\section{References}

[1] DAI, X. R., C. K. LAI, and X. G. HE: Spectral property of Cantor measures with consecutive digits. - Adv. Math. 242, 2013, 187-208.

[2] Duffin, R. J., and A. C. Schaeffer: A class of nonharmonic Fourier series. - Trans. Amer. Math. Soc. 72, 1952, 341-366.

[3] Dutkay, D. E., D. Han, Q. Sun, and E. Weber: On the Beurling dimension of exponential frames. - Adv. Math. 226:1, 2011, 285-297.

[4] Falconer, K. J.: The Hausdorff dimension of self-affine fractals. - Math. Proc. Cambridge Philos. Soc. 103:2, 1988, 339-350.

[5] FAn, A. H., K. S. LAU, and H. RAO: Relationships between different dimensions of a measure. - Monatsh. Math. 135:3, 2002, 191-201.

[6] Feng, D.-J.: Dimension of invariant measures for affine iterated function systems. arXiv:1901.01691, 2019.

[7] FU, X., and C.K. LAI: Translational absolute continuity and Fourier frames on a sum of singular measures. - J. Funct. Anal. 274:9, 2018, 2477-2498.

[8] He, X. G., Q. KAng, M. TANG, and Z. Wu: Beurling dimension and self-similar measures. J. Funct. Anal. 274:8, 2018, 2245-2264.

[9] He, X. G., C. K. LAI, and K. S. LAU: Exponential spectra in $L^{2}(\mu)$. - Appl. Comput. Harmon. Anal. 34:3, 2013, 327-338.

[10] Hochman, M., and A. RApaport: Hausdorff dimension of planar self-affine sets and measures with overlaps. - arXiv:1904.09812, 2019.

[11] Hutchinson, J. E.: Fractals and self-similarity. - Indiana Univ. Math. J. 30:5, 1981, 713-747.

[12] Iosevich, A., C. K. Laim, B. LiU, and E. Wyman: Fourier frames for surface-carried measures. - arXiv:1905.07032, 2019.

[13] ŁabA, I., and Y. WAng: Some properties of spectral measures. - Appl. Comput. Harmon. Anal. 20:1, 2006, 149-157.

[14] LAI, C. K.: On Fourier frame of absolutely continuous measures. - J. Funct. Anal. 261:10, 2011, 2877-2889.

[15] LEv, N.: Fourier frames for singular measures and pure type phenomena. - Proc. Amer. Math. Soc. 146:7, 2018, 2883-2896.

[16] SHI, R.X.: On p-adic spectral measures. - arXiv:2002.07559, 2020. 